\title{
Activation of Hepatic Stellate Cells is Inhibited by microRNA-378a-3p via Wnt10a
}

\author{
Fujun Yu ${ }^{a, b, c}$ XuFei Fan ${ }^{d}$ Bicheng Chen ${ }^{e}$ Peihong Dong ${ }^{a}$ Jianjian Zheng ${ }^{e}$
}

\begin{abstract}
aDepartment of Infectious Diseases, The First Affiliated Hospital of Wenzhou Medical University, Wenzhou, 'D Department of Gastroenterology, Songjiang Hospital Affiliated Shanghai First People's Hospital, Shanghai Jiao Tong University, Shanghai, 'Department of Gastroenterology, Shanghai Songjiang Hospital Affiliated to Nanjing Medical University, Nanjing, 'Emergency Department, The First Affiliated Hospital of Wenzhou Medical University, Wenzhou, eKey Laboratory of Surgery, The First Affiliated Hospital of Wenzhou Medical University, Wenzhou, China
\end{abstract}

\section{Key Words}

MicroRNA-378a-3p • Hepatic stellate cell $\cdot$ Wnt/ß-catenin pathway

\begin{abstract}
Background/Aims: Wnt/ $\beta$-catenin pathway is involved in liver fibrosis and microRNAs (miRNAs) are considered as key regulators of the activation of hepatic stellate cells (HSCs). A recent study showed the protective role of miR-378a-3p against cardiac fibrosis. However, whether miR-378a-3p suppresses Wnt/ $\beta$-catenin pathway in liver fibrosis is largely unknown. Methods: miR-378a-3p expression was detected in carbon tetrachloride-induced liver fibrosis and activated HSCs. Effects of miR-378a-3p overexpression on HSC activation and Wnt/ $\beta$-catenin pathway were analyzed. Bioinformatic analysis was employed to identify the potential targets of miR-378a-3p. Serum miR-378a-3p expression was analyzed in patients with cirrhosis. Results: Reduced miR-378a-3p expression was observed in the fibrotic liver tissues and activated HSCs. Up-regulation of miR-378a-3p inhibited HSC activation including cell proliferation, $\alpha$-smooth muscle actin ( $\alpha$-SMA) and collagen expression. Moreover, miR$378 a-3 p$ overexpression resulted in $\mathrm{Wnt} / \beta$-catenin pathway inactivation. Luciferase reporter assays demonstrated that Wnt10a, a member of Wnt/ $\beta$-catenin pathway, was confirmed to be a target of miR-378a-3p. By contrast, miR-378a-3p inhibitor contributed to HSC activation, with an increase in cell proliferation, $\alpha$-SMA and collagen expression. But all these effects were blocked down by silencing of Wnt10a. Notably, sera from patients with cirrhosis contained lower levels of miR-378a-3p than sera from healthy controls. Receiver operating characteristic curve analysis suggested that serum miR-378a-3p differentiated liver cirrhosis patients from healthy controls, with an area under the curve of ROC curve of 0.916. Conclusion: miR-378a$3 p$ suppresses HSC activation, at least in part, via targeting of Wnt10a, supporting its potential utility as a novel therapeutic target for liver fibrosis.

Jianjian Zheng,

and Peihong Dong,

\section{KARGER}

Key Laboratory of Surgery, the First Affiliated Hospital of Wenzhou Medical University, No.2 fuxue lane, Wenzhou 325000, Zhejiang (P.R. China) and Department of Infectious Diseases, the First Affiliated Hospital of Wenzhou Medical University, No.2 fuxue lane, Wenzhou, Zhejiang (P.R. China); E-Mail 120378196@qq.com / dongpeihong111@163.com
\end{abstract}




\section{Cellular Physiology Cell Physiol Biochem 2016;39:2409-2420 \begin{tabular}{l|l|l} 
and Biochemistry & $\begin{array}{l}\text { DOI: 10.1159/000452509 } \\
\text { Published online: November 11, } 2016\end{array}$ & $\begin{array}{l}\text { (c) } 2016 \text { The Author(s). Published by S. Karger AG, Basel } \\
\text { www.karger.com/cpb }\end{array}$
\end{tabular} \\ Yu et al.: MicroRNA-378a-3p in Hepatic Stellate Cell}

\section{Introduction}

Liver fibrosis is a chronic disorder that is characterized by an imbalance between fibrogenesis and fibrinolysis, which leads to an excessive accumulation of extracellular matrix (ECM) in liver and finally distorts the normal liver architecture [1]. Activated hepatic stellate cells (HSCs) are required for the increase in ECM production in liver [2, 3]. During HSC activation, quiescent HSCs lose cytoplasmic lipid droplets and trans-differentiate to proliferative, fibrogenic myofibroblasts [4]. However, to date, the mechanism of HSC activation is still not completely understood.

MiRNAs are endogenous small ( $\sim 22 \mathrm{nt})$ non-coding RNAs molecules found in a variety of eukaryotic organisms, which control gene expression by interacting with the 3 '-untranslated region (3'-UTR) of target gene mRNA to repress translation or enhance mRNA cleavage [5]. miRNAs are implicated in a wide variety of biological processes including cell proliferation, apoptosis, metabolism, differentiation, immune response and development [6-8]. Over the past several years, gene array analysis of miRNA expression in carbon tetrachloride $\left(\mathrm{CCl}_{4}\right)$ induced hepatic fibrogenesis model has identified a panel of deregulated miRNAs [9]. Among them, many miRNAs have been revealed to regulate transforming growth factor- $\beta$ (TGF $\beta$ ) signaling. For example, miR-29b inhibits HSC activation through TGF $\beta 1$ and Smad3 [10]. HSC activation involves many signaling pathways such as TGF $\beta$ /Smads signaling and Wnt/ $\beta$-catenin pathway. Previously, we demonstrated that miR-17-5p activates HSCs through targeting of Smad7 and miRNA-125a-5p contributes to HSC activation through targeting hypoxia-inducible factor $1[7,11]$. However, there are few reports about the regulation of Wnt/ $\beta$-catenin pathway by miRNAs in liver fibrosis.

Recently, miR-378a-3p has been reported to be down-regulated in various diseases including cancers [12-14]. Growing evidence has demonstrated that miR-378a-3p is involved in a wide range of biological functions and processes such as cell growth, cell cycle, migration, differentiation, metabolism and angiogenesis $[12,15]$. A recent study shows that miR-378a$3 p$, a negative regulator of TGF $\beta 1$, contributes to the suppression of cardiac fibrosis [16]. Hyun et al. found that miR-378a-3p could suppress HSC activation by targeting Gli3 [17]. However, the biological role of miR-378a-3p in liver fibrosis remains largely unclear. In this study, we found that miR-378a-3p expression was reduced in fibrotic liver tissues and activated HSCs. Overexpression of miR-378a-3p resulted in the suppression of HSC activation including HSC proliferation, $\alpha$-smooth muscle actin ( $\alpha$-SMA) and type I collagen. We demonstrated that miR-378a-3p inhibits HSC activation, at least in part, via targeting of Wnt10a.

\section{Materials and Methods}

\section{Clinical Samples}

Serum samples of 15 patients with cirrhosis and 15 healthy controls obtained between 2011 and 2012 were collected at the First Affiliated Hospital of Wenzhou Medical University, Wenzhou, China (Table 1). Informed consent for use of blood samples was obtained from all participants, and the project approved by the Ethics Committee of the First Affiliated Hospital of Wenzhou Medical University.

\section{Rat liver fibrosis model}

Adult male Sprague-Dawley (180-220 g) received an intraperitoneal injection of $2 \mathrm{ml}$ of $\mathrm{CCl}_{4} /$ olive oil $(1: 1, \mathrm{v} / \mathrm{v}) / \mathrm{kg}$ body weight to generate liver fibrosis [18]. Moreover, $\mathrm{CCl}_{4}$ was given twice a week for 6 weeks. Control rats were treated with an equal volume of olive oil at the same time intervals. Rats were provided by the Experimental Animal Center of Wenzhou Medical University and the experimental protocol was approved by the Institutional Animal Committee of Wenzhou Medical College. All animals received care in accordance with 'Guide for the Care and Use of Laboratory Animals'. Rats were sacrificed under anesthesia at the end of six weeks and the livers were removed for further analysis. The liver tissues were used for hematoxylin and eosin (H\&E) staining and Masson staining by fixation with $10 \%$ formalin. 


\section{Cellular Physiology Cell Physiol Biochem 2016;39:2409-2420

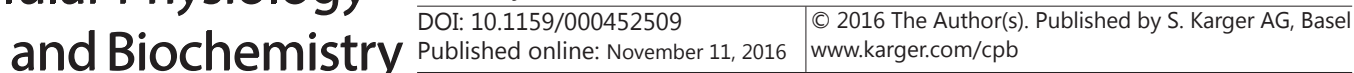 \\ Yu et al.: MicroRNA-378a-3p in Hepatic Stellate Cell}

Table 1. Human subjects

\begin{tabular}{|c|c|c|}
\hline Parameters & $\begin{array}{c}\text { Patients } \\
15\end{array}$ & $\begin{array}{c}\text { Controls } \\
15\end{array}$ \\
\hline \multicolumn{3}{|l|}{ Sex, n (\%) } \\
\hline Male & $9(60.0 \%)$ & $8(53.3 \%)$ \\
\hline Female & $6(40.0 \%)$ & $7(46.7 \%)$ \\
\hline Age, $n( \pm S D)$ & $46.1( \pm 4.2)$ & $45.3( \pm 6.4)$ \\
\hline \multicolumn{3}{|l|}{ Etiology, n (\%) } \\
\hline $\mathrm{HBV}$ & $15(100 \%)$ & - \\
\hline Serum ALT, U/L & $99.3( \pm 37.2)$ & $32.7( \pm 9.1)$ \\
\hline Serum AST, U/L & $113.2( \pm 21.4)$ & $24.8( \pm 4.7)$ \\
\hline \multicolumn{3}{|l|}{ Child-Pugh stage } \\
\hline $\mathrm{A}$ & $7(46.7 \%)$ & - \\
\hline B & $6(40.0 \%)$ & - \\
\hline $\mathrm{C}$ & $2(13.3 \%)$ & - \\
\hline
\end{tabular}

Cell culture and transfection

Adult male Sprague-Dawley rats were used for HSC isolation as described previously [19]. The purity of cultures was confirmed by immunocytochemical staining for $\alpha$-SMA and the purity reached $>98 \%$. Hepatocytes were isolated using a two-step collagenase perfusion technique [20]. Then, gene expression levels including F4/80, CD32b and CYP3A11 were measured by quantitative real-time PCR (qRT-PCR). Hepatocyte purity was found to be greater than $95 \%$. Chemically synthesized RNAs including negative control (miR-NC), miR-378a-3p mimics and miR-378a-3p inhibitor were obtained from GenePharma biotechnology (Shanghai, China). For transfection, HSCs were transfected with $1 \mu \mathrm{g}$ of the chemically synthesized RNA using Lipofectamine 2000 (Invitrogen, USA).

\section{RNA interference analysis}

RNA interference experiments were performed using Lipofectamine 2000 (Invitrogen) in accordance with the manufacturer's instructions. Wnt10a siRNA (sense 5'- UAAUGACGCCCAUUUCUGCGU-3'; antisense $5^{\prime}$-GCAGAAAUGGGCGUCAUU AGG-3') and scrambled siRNA (negative control) were designed and synthesized by GenePharma biotechnology. siRNAs were transfected into cells at a final concentration of $100 \mathrm{nM}$.

\section{Cell proliferation assay}

Cell proliferation was assessed using CCK-8 (Dojindo, Kumamoto, Japan) according to manufacturer's instructions. Cells were seeded in 96-well plates at a density of $1 \times 10^{3}$ cells per well and cultured for $24 \mathrm{~h}$. Next, cells were transfected with miR-378a-3p mimics. After 48 h, CCK-8 solution was added to each well in the 96 -well plates. Then, cells were incubated for additional $2 \mathrm{~h}$ at $37^{\circ} \mathrm{C}$. Absorbance was determined at 450 $\mathrm{nm}$ on a microplate reader (Molecular Devices, Sunnyvale, CA, USA).

\section{Cell cycle analysis}

The Cell Cycle Analysis Kit (Beyotime, China) was used to analyze cell cycle. Cells were fixed in $70 \%$ ethanol in PBS at $-20^{\circ} \mathrm{C}$ for $24 \mathrm{~h}$ and then labeled with $0.5 \mathrm{ml}$ propidium iodide (PI) staining buffer containing $200 \mathrm{mg} / \mathrm{ml} \mathrm{RNase}$ A and $50 \mu \mathrm{g} / \mathrm{ml} \mathrm{PI}$ at $37^{\circ} \mathrm{C}$ for $40 \mathrm{~min}$ in the dark. Analyses were performed on a BD LSR flow cytometer (BD Biosciences).

qRT-PCR

Total RNA was extracted from serum, rat liver tissues and cells using miRNeasy Mini kit (Qiagen, Valencia, CA, USA) according to manufacturer's instructions. Gene expression was measured by real-time PCR using SYBR Green real-time PCR Master Mix (Toyobo, Osaka, Japan). The primers of alpha-1 (I) collagen (Col1A1), $\alpha$-SMA, and GAPDH were designed as described previously [21]. The primers used for Wnt10a were 5'-GATGGTGGGGCATCGTGAA-3' and 5'-GGGTTCTGTCGGATCAGTCG-3'. Expressions of miR-378a-3p and miR-16 were detected using the TaqMan MicroRNA Assay (Applied Biosystems, Foster City, CA). The GAPDH level was used to normalize the relative abundance of mRNAs. miR-16, an ubiquitous miRNA which is not specific for liver, was used to normalize the relative abundance of miR-378a-3p [22]. The expression levels (2- $\left.{ }^{-\triangle A t}\right)$ of mRNAs and miR-378a-3p were calculated as described previously [23]. 


\section{Cellular Physiology Cell Physiol Biochem 2016;39:2409-2420

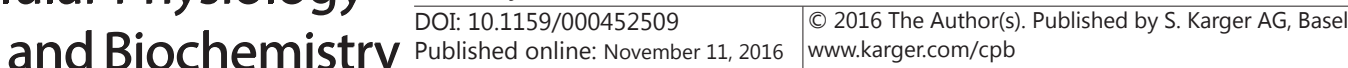 \\ Yu et al.: MicroRNA-378a-3p in Hepatic Stellate Cell}

Western blot analysis

For assessing sample protein concentration, we performed a BCA protein assay kit (Beyotime Biotechnology, Jiangsu, China). Total proteins (30-50 $\mu \mathrm{g}$ ) were separated by SDS-PAGE and transferred to a PVDF membrane (Millipore Corp, Billerica, MA, USA). After blocking, the membranes were incubated for $1 \mathrm{~h}$ with primary antibodies diluted in TBS/Tween20 (0.075\%) containing 3\% Marvel. Rabbit polyclonal antibodies against type I collagen, Wnt10a and phosphorylated $\beta$-catenin (Y86) were diluted 1:2000, and mouse monoclonal antibodies against $\alpha$-SMA, glycogen synthase kinase-3 $\beta$ (GSK-3 $\beta$ ) and GAPDH were used at 1:4000. The membranes were washed 3 times with TBS/Tween20 (0.075\%) containing 3\% Marvel, followed by incubation with HRP-conjugated secondary antibodies $(1: 5000)$ at $37^{\circ} \mathrm{C}$ for $1 \mathrm{~h}$. The antigenantibody complex was developed by enhanced chemiluminescence, exposed in the dark room and analyzed for integral absorbance (IA) of the protein bands using quantitative software, Quantity One 4.4.

\section{Immunofluorescence microscopy}

HSCs were seeded on 18-mm cover glasses and fixed in an acetic acid: ethanol (1:3) solution for 5 $\min$ at $-20^{\circ} \mathrm{C}$. Nonspecific binding was blocked with $5 \%$ goat serum/PBS for $1 \mathrm{~h}$ at room temperature. Then, cells were incubated with primary antibodies against $\alpha$-SMA, type I collagen or $\beta$-catenin (Abcam), followed by fluorescein-labeled secondary antibody (1:50 dilution; Dianova). The nuclei were stained with 4,6-diamidino-2-phenylindole (DAPI). The slides were washed twice with PBS, covered with DABCO (SigmaAldrich), and examined with confocal laser scanning microscopy (Olympus, Tokyo, Japan) at 488 and 568 nm.

Luciferase activity assay

HSC-T6 cells, obtained from Research of the Chinese Academy of Medical Sciences (Beijing, China), were co-transfected with either luciferase reporter plasmid harboring wild-type Wnt10a 3'UTR (pmirGLOWnt10a-wt) or mutant Wnt10a 3'UTR (pmirGLO-Wnt10a-mut) together with miR-378a-3p mimics or miRNC. The 3'UTR of Wnt10a was cloned downstream of the firefly luciferase gene. Whether the activity of firefly luciferase is down-regulated or not is depended on the interaction between miR-378a-3p and the 3'UTR of Wnt10a mRNA. Approximately $48 \mathrm{~h}$ after transfection, the cells were harvested and luciferase activity was determined by a luminometer using a Dual-Luciferase Reporter Assay System (Promega, Madison, WI, USA).

TCF reporter activity assay

Cells were transiently transfected with TOPFLASH and FOPFLASH (Upstate Biotechnology Inc., Lake Placid, NY, USA) using Lipofectamine 2000. Twenty four hours after transfection, the cells were harvested and luciferase and Renilla luminescence were measured using the Dual-Luciferase Reporter Assay System (Promega, Wisconsin, WI, USA) on a luminometer (BioTek Instruments, Winooski, VT, USA). TCF reporter activity was presented as the ratio of firefly-to-Renilla luciferase activity.

\section{Statistical analysis}

Data from at least three independent experiments were expressed as the mean $\pm \mathrm{SD}$. Comparisons between two groups and multiple groups were made using Student's $t$-test and one-way analysis of variance, respectively. Receiver operating characteristic (ROC) curve was generated to evaluate the diagnostic potential of serum miR-378a-3p via calculation of the area under the ROC curve (AUC), sensitivity and specificity according to standard formulas. $P<0.05$ was considered significant. All statistical analyses were performed with SPSS software (version 13; SPSS, Chicago, IL).

\section{Results}

miR-378a-3p is reduced in liver fibrotic tissues and activated HSCs

The excessive accumulation of collagens is a major pathogenic feature of fibrotic diseases. $\mathrm{CCl}_{4}$, which is the most commonly used hepatotoxic reagent, was used to generate rat liver fibrosis. Liver fibrosis in rats was established via injection of $\mathrm{CCl}_{4}$ for 6 weeks, and confirmed by H\&E and Masson staining (Fig. 1A). Next, miR-378a-3p expression was detected in liver tissues from $\mathrm{CCl}_{4}$-treated rats. qRT-PCR analysis showed that miR-378a-3p 
Fig. 1. miR-378a-3p expression in $\mathrm{CCl}_{4}$-treated hepatic fibrotic tissues and primary HSCs. (A) Hepatic fibrotic and control tissues $(n=6)$ were detected by $\mathrm{H} \& \mathrm{E}$ staining $(\times 100)$ and Masson staining $(\times 100)$, respectively. (B) miR378a-3p expression was detected in fibrotic liver tissues and control liver tissues $(n=6)$. (C) Compared with healthy controls, miR-378a-3p expression was decreased in HSCs and hepatocytes isolated from $\mathrm{CCl}_{4}$-rats. (D) In healthy rats, miR378a-3p expression was significantly higher in primary HSCs compared with that in primary hepatocytes. After $\mathrm{CCl}_{4}$ treatment, miR-378a$3 p$ expression was reduced in primary HSCs compared with primary hepatocytes. (E) miR378a-3p expression was detected in primary HSCs during different culture days. Each value is the mean $\pm \mathrm{SD}$ of three experiments. $\quad * P<0.05$ compared with the control or primary hepatocytes.
A
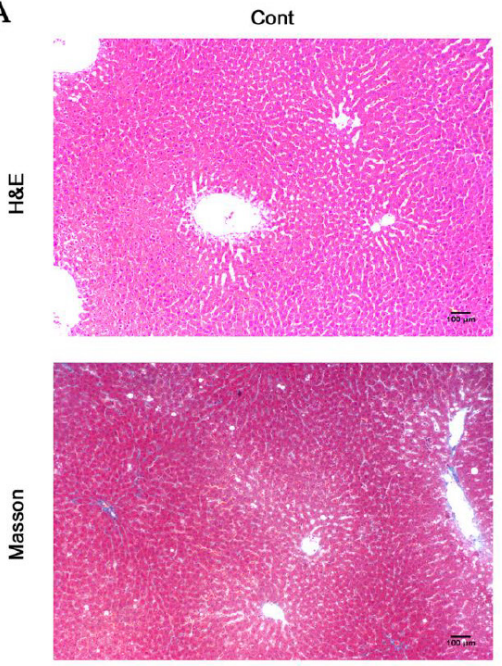

B

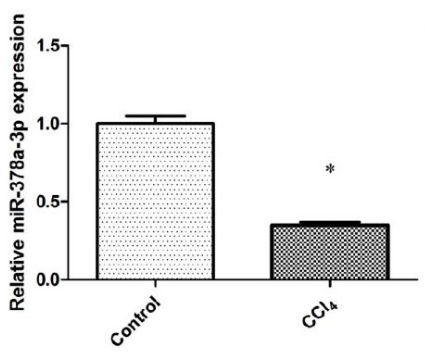

D

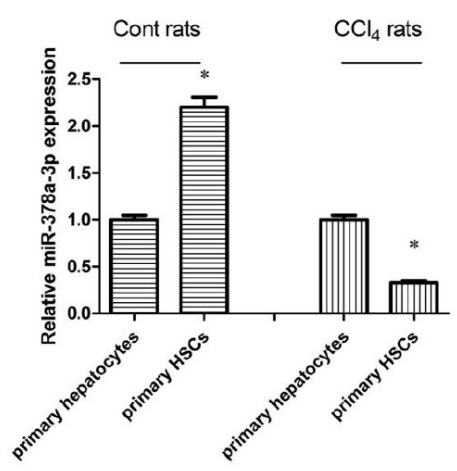

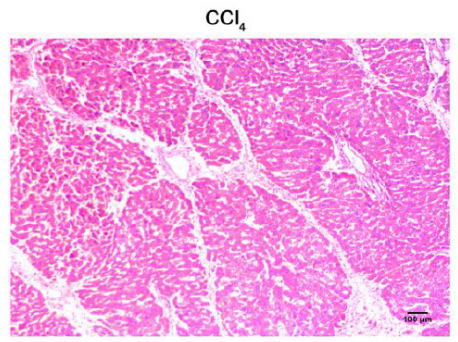

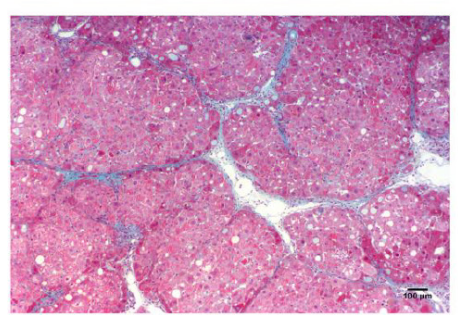

$\mathrm{C}$

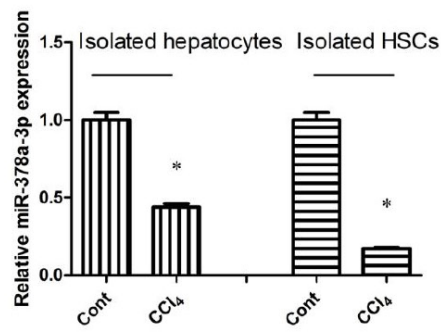

E

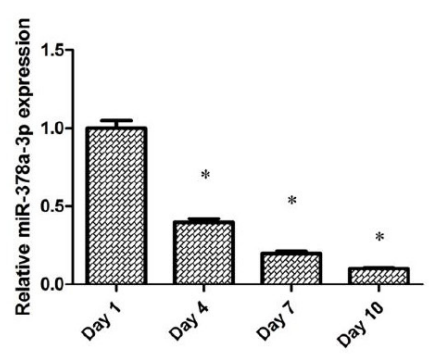

was significantly down-regulated in $\mathrm{CCl}_{4}$-treated liver relative to the control liver (Fig.1B). Further study showed that compared with healthy controls, miR-378a-3p expression was decreased by $83 \%$ and $56 \%$ in $\mathrm{HSCs}$ and hepatocytes isolated from $\mathrm{CCl}_{4}$-rats, respectively (Fig.1C). In healthy rats, miR-378a-3p expression was significantly higher in primary HSCs compared with that in primary hepatocytes (Fig.1D). After $\mathrm{CCl}_{4}$ treatment, miR-378a$3 p$ expression was significantly lower in primary HSCs than that in primary hepatocytes (Fig.1D). Combined these, the expression and role of miR-378a-3p were further explored in activated HSCs. Freshly isolated HSCs are known to lose their quiescent phenotype and gradually acquire myofibroblastic phenotype during culture days. Therefore, HSCs were isolated from the livers of healthy rats and cultured for up to 10 days. Interestingly, with time in culture, there was a significant reduction in miR-378a-3p expression (Fig.1E). These data suggest that reduced miR-378a-3p level may be associated with the progression of liver fibrosis. 


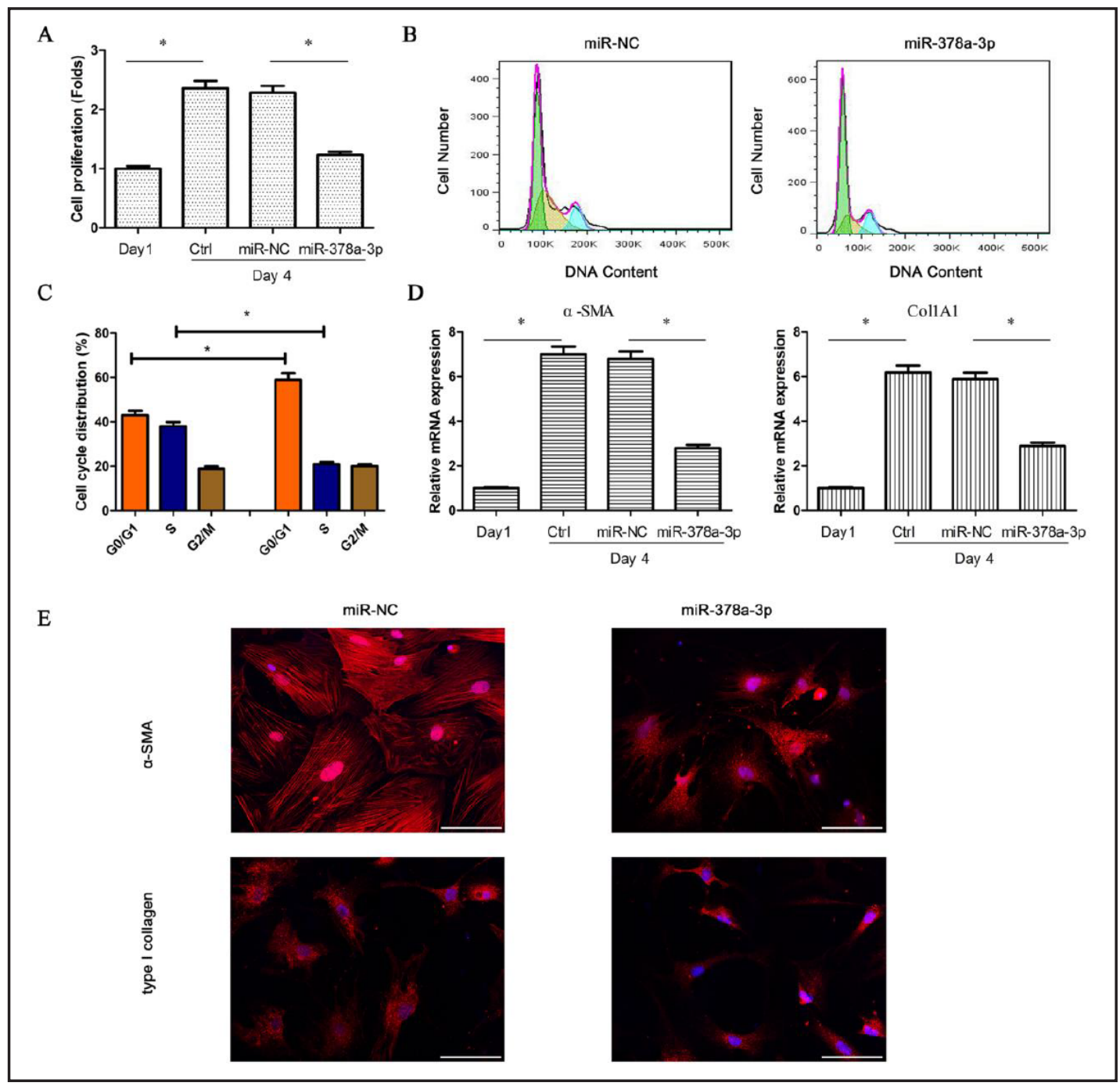

Fig. 2. Effects of miR-378a-3p overexpression on cell proliferation, cell cycle, HSC transdifferentiation and collagen expression. Primary 4-day-old HSCs were transfected with miR-378a-3p mimics for 48 h. (A) Enhanced cell proliferation in Day 4 was inhibited by miR-378a-3p. (B and C) miR-378a-3p suppressed cell cycle. (D) Increased mRNA expressions of $\alpha$-SMA and Col1A1 in Day 4 were inhibited by miR-378a-3p. (E) Immunofluorescence staining for $\alpha$-SMA (red) and type I collagen (red) were evaluated by confocal laser microscopy. DAPI stained nuclei blue. Scale bar, $100 \mu \mathrm{m}$. Each value is the mean \pm SD of three experiments. $* P<0.05$.

\section{Overexpression of miR-378a-3p suppresses HSC activation}

To explore whether miR-378a-3p overexpression suppresses HSC activation, primary HSCs were transfected with miR-378a-3p mimics. As shown by the results of CCK-8 assays, HSC proliferation was increased in Day 4 compared with Day 1 (Fig. 2A). Notably, increased cell proliferation in Day 4 was inhibited by miR-378a-3p mimics (Fig. 2A). Further study showed that miR-378a-3p increased a proportion of cells in the G0/G1 phase and reduced the number of cells in the $\mathrm{S}$ phase, as indicated by cell cycle analysis (Fig. 2B and Fig. 2C). Next, we investigated the effects of miR-378a-3p overexpression on HSC transdifferentiation and collagen expression. Compared with Day 1, the mRNA expressions of $\alpha$-SMA and Col1A1 were enhanced in Day 4 (Fig. 2D). But the increased $\alpha$-SMA and Col1A1 mRNA expressions in Day 4 were suppressed by miR-378a-3p mimics (Fig. 2D). Further study demonstrated

\section{KARGER}




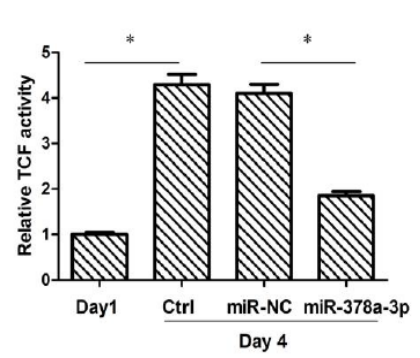

C

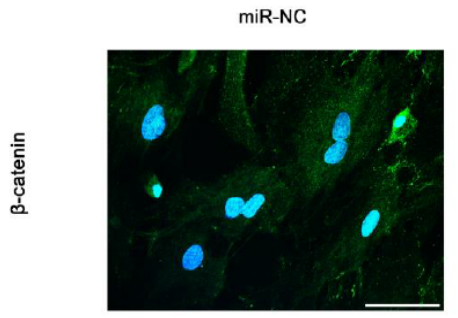

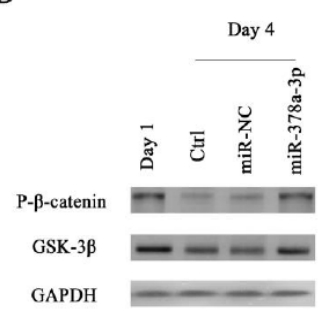

$\operatorname{miR}-378 a-3 p$

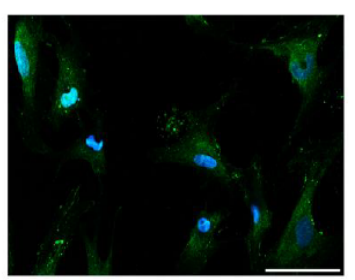

Fig. 3. Wnt/ $\beta$-catenin pathway is involved in the effects of miR-378a-3p on HSC activation. Primary 4-dayold HSCs were transfected with miR-378a-3p mimics for 48 h. (A) Enhanced TCF activity in Day 4 was reduced by miR-378a-3p. (B) Reduced P- $\beta$-catenin and GSK-3 $\beta$ protein in Day 4 were restored by miR-378a3 p. GAPDH was used as internal control. (C) Immunofluorescence staining for $\beta$-catenin (green) was evaluated by confocal laser microscopy. DAPI stained nuclei blue. Scale bar, $100 \mu \mathrm{m}$. Each value is the mean \pm SD of three experiments. ${ }^{*} P<0.05$.

that there was a reduction in $\alpha$-SMA fibers in miR-378a-3p mimics group, as indicated by immunofluorescence analysis (Fig. 2E). Likewise, miR-378a-3p induced a decrease in collagen expression (Fig. 2E). Taken together, overexpression of miR-378a-3p contributes to the suppression of HSC activation.

Wht/ $\beta$-catenin pathway is involved in the effects of miR-378a-3p in liver fibrosis

We next determined whether Wnt/ $\beta$-catenin pathway is involved in the effects of miR$378 a-3 p$ on liver fibrosis. As indicated by the results of TCF reporter activity assay, TCF activity was increased in Day 4 compared with Day 1 (Fig. 3A). Interestingly, increased TCF activity in Day 4 was inhibited by miR-378a-3p overexpression (Fig. 3A). Consistent with the reduced TCF activity, reduced P- $\beta$-catenin protein level in Day 4 was restored by miR378a-3p (Fig. 3B). Further study showed that there was a reduction in $\beta$-catenin protein in cells transfected with miR-378a-3p mimics, as indicated by immunofluorescence analysis (Fig. 3C). GSK-3 $\beta$, a key element of $\beta$-catenin/Wnt pathway, was also examined in miR-378a$3 p$ overexpression cells. We found that GSK-3 $\beta$ protein expression was reduced in Day 4 compared with Day 1 (Fig. 3B). But reduced GSK-3 $\beta$ protein expression in Day 4 was restored by miR-378a-3p (Fig. 3B). Collectively, these data suggest that miR-378a-3p suppresses HSC activation, at least in part, via Wnt/ $\beta$-catenin pathway.

Wnt10a is a target of miR-378a-3p and involved in the effects of miR-378a-3p on HSC activation

To determine the mechanism by which miR-378a-3p inhibits HSC activation, bioinformatic analysis (miRDB) was employed to identify the potential targets of miR$378 \mathrm{a}-3 \mathrm{p}$. Wnt10a, a component of Wnt/ $\beta$-catenin pathway, predicted as a putative target of miR-378a-3p (Fig. 4A). Next, the 3'UTR target sequence of Wnt10a mRNA was cloned into the pmirGLO plasmid to confirm whether miR-378a-3p could directly regulate Wnt10a expression via the predicted binding site. In miR-378a-3p transfected cells, there was a 
A

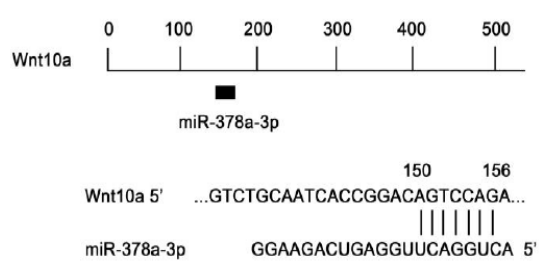

$\mathrm{C}$

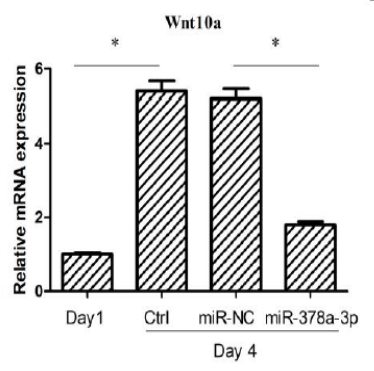

D
B
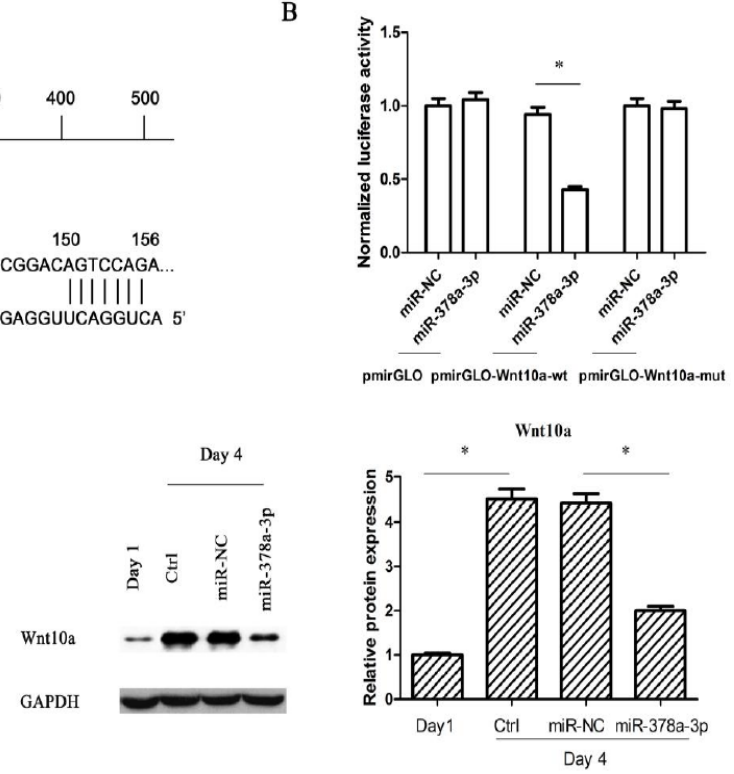

Fig. 4. Interaction between miR-378a-3p and Wnt10a 3'UTR. Primary 4-day-old HSCs were transfected with miR-378a-3p mimics for 48 h. (A) miR-378a-3p-binding sites in the 3'UTR of Wnt10a mRNA based on miRDB software. (B) Analysis of luciferase activity in HSC-T6 cells transfected with pmirGLO-Wnt10a-wt or pmirGLO-Wnt10a-mut as well as those co-transfected with miR-378a-3p mimics or miR-NC $(n=3)$. (C) Wnt10a mRNA expression was enhanced in Day 4, which was reduced by miR-378a-3p. (D) Increased Wnt10a protein expression in Day 4 was inhibited by miR-378a-3p. Each value is the mean \pm SD of three experiments. ${ }^{*} P<0.05$.

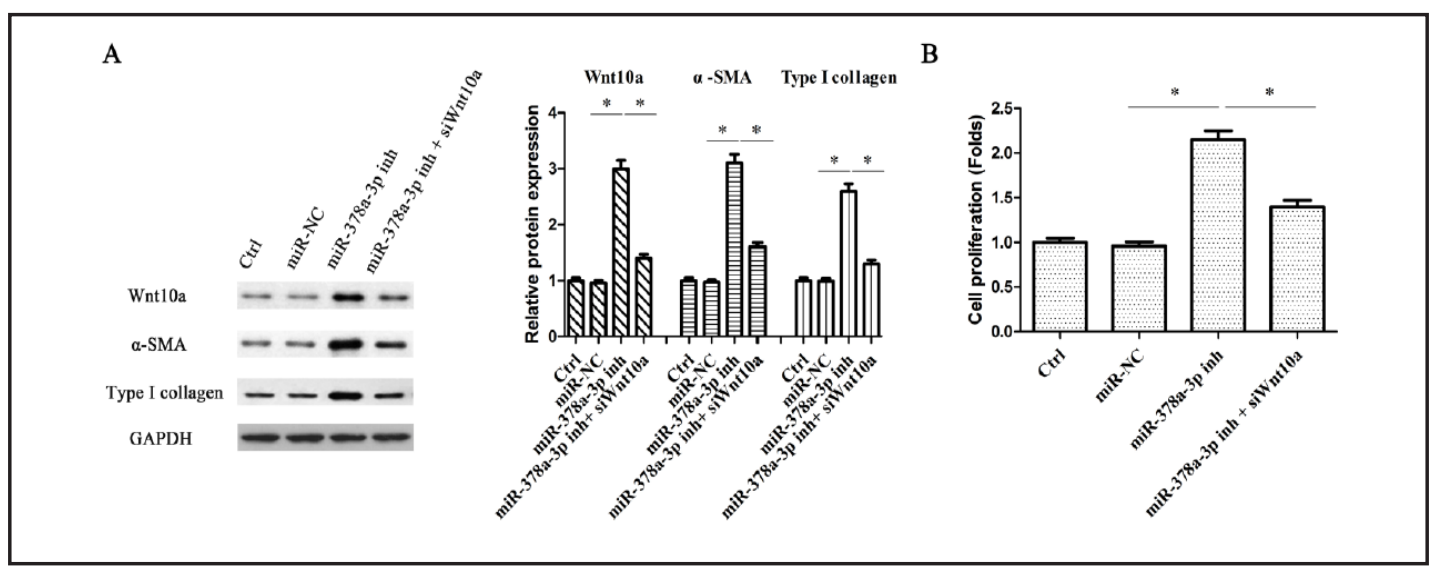

Fig. 5. Effects of miR-378a-3p inhibitor on HSC activation are inhibited by silencing of Wnt10a. Primary 4-day-old HSCs were transfected with miR-378a-3p inhibitor for $48 \mathrm{~h}$ and then treated with siWnt10a for additional $48 \mathrm{~h}$. (A) Increased protein levels of Wnt10a, $\alpha$-SMA and type I collagen induced by miR-378a-3p inhibitor were blocked down by siWnt10a. (B) miR-378a-3p inhibitor-induced cell proliferation was inhibited by siWnt10a. ${ }^{*} P<0.05$.

significant reduction in luciferase activity in pmirGLO-Wnt10a-wt, whereas the luciferase activity was not altered in pmirGLO-Wnt10a-mut (Fig. 4B). To validate these results, the mRNA and protein expressions of Wnt10a were examined in miR-378a-3p transfected cells. It was found that increased Wnt10a mRNA and protein levels in Day 4 were blocked down by miR-378a-3p (Fig. 4C and Fig. 4D). All these data suggest that Wnt10a is a target of miR-378a- 
A

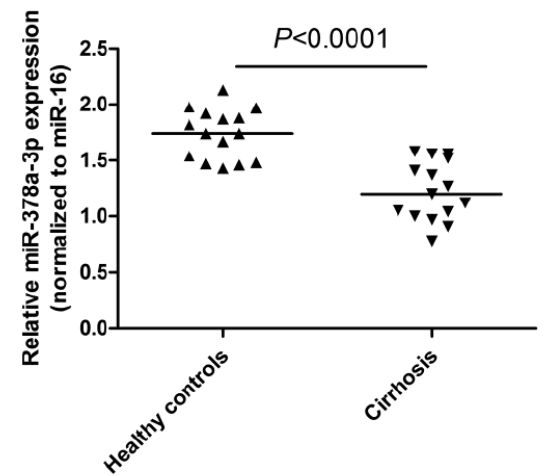

B

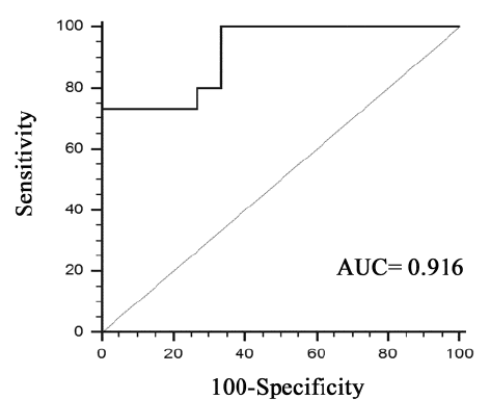

Fig. 6. miR-378a-3p expression in serum of patients with liver cirrhosis. (A) Serum levels of miR-378a-3p were significantly down-regulated in patients with cirrhosis, compared to healthy controls $(P<0.0001)$. (B) ROC curve analysis of serum miR-378a-3p for discriminating patients with cirrhosis from healthy controls.

3p. To further confirm whether Wnt10a was involved in the effects of miR-378a-3p on HSC activation, primary HSCs were transfected with miR-378a-3p inhibitor and then treated with Wnt10a siRNA (siWnt10a). We found that miR-378a-3p inhibitor resulted in a significant increase in Wnt10a protein expression (Fig. 5A). Notably, miR-378a-3p inhibitor contributed to HSC activation, with an increase in $\alpha$-SMA, type I collagen and cell proliferation (Fig. $5 \mathrm{~A}$ and Fig. 5B). But these effects were blocked down by silencing of Wnt10a (Fig. 5A and Fig. 5B), suggesting that miR-378a-3p inhibits HSC activation, at least in part, via Wnt10a.

miR-378a-3p is reduced in serum of patients with liver cirrhosis

Increasing evidence suggests that serum miRNAs are potential biomarkers for various diseases [24, 25]. A total of 30 subjects were recruited, including 15 patients with liver cirrhosis and 15 healthy controls (Table 1). In the present study, there were no significant differences in age between patients with liver cirrhosis and healthy controls $(P=0.676)$. Moreover, no significant difference was observed in sex distribution $\left(P=0.713, \chi^{2}\right.$ test $)$. To investigate whether aberrantly expressed miR-378a-3p is present in patients with liver cirrhosis, qRT-PCR analysis was performed to detect miR-378a-3p levels in sera from patients with liver cirrhosis as well as healthy controls. Interestingly, serum miR-378a-3p levels were significantly down-regulated in patients with cirrhosis compared to healthy controls (Fig. $6 \mathrm{~A})$. To further investigate whether serum miR-378a-3p level could serve as a potential diagnostic marker for liver fibrosis, ROC curve analysis was performed. It was found that serum miR-378a-3p differentiated liver cirrhosis patients from healthy controls, with an AUC of ROC curve of 0.916 [95\% confidence interval (CI), 0.755 to 0.985] (Fig. 6B). At the cutoff value of 1.41 , the sensitivity and the specificity were $73.3 \%$ and $100 \%$, respectively.

\section{Discussion}

Activation of HSC is a crucial event in the development of liver fibrosis. Generally, HSC activation is characterized by the accumulation of collagens, the enhancement of $\alpha$-SMA expression and the increase of cell proliferation [26]. In this study, miR-378a-3p overexpression contributes to the suppression of $\alpha$-SMA and collagen expressions. Moreover, miR-378a-3p overexpression inhibited HSC proliferation as well as cell cycle. All these data suggest that miR-378a-3p overexpression inhibits the activation of HSC.

A growing body of data shows a biological role of miR-378a-3p in a variety of diseases [27, 28]. In cancer, miR-378a suppresses the growth of hepatitis $B$ virus-related hepatocellular carcinoma by targeting the insulin-like growth factor 1 receptor [27]. In adipogenesis and 
obesity, miR-378a-3p has been reported to enhance adipogenesis by targeting mitogenactivated protein kinase 1 [29]. In addition, reduced miR-378a-3p by flavonoid fisetin inhibited fat accumulation in the liver [30]. In organ fibrosis, miR-378a-3p has been reported to be abundant in cardiomyocytes and miR-378a-3p down-regulation contributes to the development of cardiac fibrosis involving a TGF 31 -dependent paracrine mechanism [16, 31]. In this study, we found that miR-378a-3p was down-regulated in $\mathrm{CCl}_{4}$-treated rats and activated HSCs. Of note, overexpression of miR-378a-3p contributes to the suppression of activated HSCs, with a reduction in HSC activation markers including $\alpha$-SMA and Col1A1. Overexpression of miR-378a-3p additionally led to a reduction in HSC proliferation. By contrast, miR-378a-3p inhibitor resulted in HSC activation. These data suggest that miR378a may serve as a potential therapeutic target for liver fibrosis. In addition, recent studies show that serum miR-378a-3p can be detectable and changed in many cancers such as prostate cancer [32], renal cancer [33] and gastric cancer [34], suggesting that serum miR378a-3p may be considered as a biomarker. Here we report that miR-378a-3p was reduced in serum of cirrhosis patients compared with healthy controls. ROC analysis suggests a potential diagnostic value of serum miR-378a-3p for liver fibrosis. However, the sample size was small, and experiments with large sample cohorts are needed to further validate the utility of this marker.

Wnt/ $\beta$-catenin, an evolutionarily conserved cellular signaling system, plays an essential role in diverse biologic processes including organ fibrosis $[35,36]$. Emerging studies show that aberrant Wnt/ $\beta$-catenin signaling is involved in liver fibrosis [37]. Aberrant activation of Wnt/ $\beta$-catenin pathway accelerates HSC activation including cell proliferation and ECM accumulation $[38,39]$. Antagonizing $W n t / \beta$-catenin signaling by Wnt inhibitors can attenuate the progression of liver fibrosis [40]. Therefore, $\mathrm{Wnt} / \beta$-catenin pathway may be a therapeutic target in liver fibrosis. In this study, reduced Wnt/ $\beta$-catenin pathway was found in miR-378a-3p transfected cells. Bioinformatics analysis and luciferase reporter experiments demonstrated that Wnt10a, a member of Wnt/ $\beta$-catenin pathway, is a target of miR-378a-3p. In addition, the mRNA and protein expressions of Wnt10a were decreased in miR-378a-3p transfected cells. Notably, the effects of miR-378a-3p inhibitor on HSC activation could be blocked down by the loss of Wnt10a. Collectively, our data suggest that miR-378a-3p inhibits HSC activation, at least in part, via suppressing Wnt10a, and this is a first report to show miR-378a-3p-mediated Wnt/ $\beta$-catenin pathway in liver fibrosis. However, the functional role of miR-378a-3p in vivo still needs further investigation.

In conclusion, we demonstrate that miR-378a-3p can inhibit HSC activation, at least in part, via targeting of Wnt10a. Serum miR-378a-3p may serve as a potential diagnostic marker for liver fibrosis.

\section{Acknowledgements}

The project was supported by the National Natural Science Foundation of China (No. 81500458/H0317), Zhejiang Provincial Natural Science Foundation of China (No. LY16H030012) and Wenzhou Municipal Science and technology Bureau (No. Y20150091).

\section{Disclosure Statement}

None.

\section{References}

1 Jiao J, Friedman SL, Aloman C: Hepatic fibrosis. Curr Opin Gastroenterol 2009;25:223-229.

2 Zhang X, Tan Z, Wang Y, Tang J, Jiang R, Hou J, Zhuo H, Wang X, Ji J, Qin X, Sun B: PTPRO-associated hepatic stellate cell activation plays a critical role in liver fibrosis. Cell Physiol Biochem 2015;35:885-898. 


\section{Cellular Physiology Cell Physiol Biochem 2016;39:2409-2420

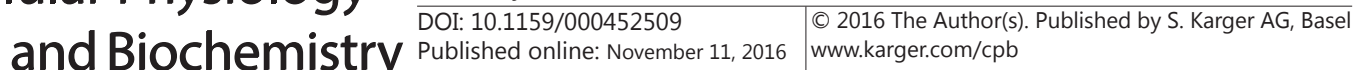 \\ Yu et al.: MicroRNA-378a-3p in Hepatic Stellate Cell}

3 Xiao Y, Qu C, Ge W, Wang B, Wu J, Xu L, Chen Y: Depletion of thymosin beta4 promotes the proliferation, migration, and activation of human hepatic stellate cells. Cell Physiol Biochem 2014;34:356-367.

$4 \quad$ Friedman SL: Liver fibrosis -- from bench to bedside. J Hepatol 2003;38 Suppl 1:S38-53.

5 Bartel DP: MicroRNAs: genomics, biogenesis, mechanism, and function. Cell 2004;116:281-297.

6 Schickel R, Boyerinas B, Park SM, Peter ME: MicroRNAs: key players in the immune system, differentiation, tumorigenesis and cell death. Oncogene 2008;27:5959-5974.

$7 \quad$ Li G, Li J, Li C, Qi H, Dong P, Zheng J, Yu F: MicroRNA-125a-5p Contributes to Hepatic Stellate Cell Activation through Targeting FIH1. Cell Physiol Biochem 2016;38:1544-1552.

8 Gong XH, Chen C, Hou P, Zhu SC, Wu CQ Song CL, Ni W, Hu JF, Yao DK, Kang JH, Zhu L: Overexpression of miR-126 inhibits the activation and migration of HSCs through targeting CRK. Cell Physiol Biochem 2014;33:97-106.

9 Roderburg C, Urban GW, Bettermann K, Vucur M, Zimmermann H, Schmidt S, Janssen J, Koppe C, Knolle P, Castoldi M, Tacke F, Trautwein C, Luedde T: Micro-RNA profiling reveals a role for miR-29 in human and murine liver fibrosis. Hepatology 2011;53:209-218.

10 Liang C, Bu S, Fan X: Suppressive effect of microRNA-29b on hepatic stellate cell activation and its crosstalk with TGF-beta1/Smad3. Cell Biochem Funct 2016;34:326-333.

11 Yu F, Guo Y, Chen B, Dong P, Zheng J: MicroRNA-17-5p activates hepatic stellate cells through targeting of Smad7. Lab Invest 2015;95:781-789.

12 Megiorni F, Cialfi S, McDowell HP, Felsani A, Camero S, Guffanti A, Pizer B, Clerico A, De Grazia A, Pizzuti A, Moles A, Dominici C: Deep Sequencing the microRNA profile in rhabdomyosarcoma reveals downregulation of miR-378 family members. BMC Cancer 2014;14:880.

13 Ikeda K, Horie-Inoue K, Ueno T, Suzuki T, Sato W, Shigekawa T, Osaki A, Saeki T, Berezikov E, Mano H, Inoue S: miR-378a-3p modulates tamoxifen sensitivity in breast cancer MCF-7 cells through targeting GOLT1A. Sci Rep 2015;5:13170.

14 Fang J, Song XW, Tian J, Chen HY, Li DF, Wang JF, Ren AJ, Yuan WJ, Lin L: Overexpression of microRNA-378 attenuates ischemia-induced apoptosis by inhibiting caspase-3 expression in cardiac myocytes. Apoptosis 2012;17:410-423.

15 Krist B, Florczyk U, Pietraszek-Gremplewicz K, Jozkowicz A, Dulak J: The Role of miR-378a in Metabolism, Angiogenesis, and Muscle Biology. Int J Endocrinol 2015;2015:281756.

16 Nagalingam RS, Sundaresan NR, Noor M, Gupta MP, Solaro RJ, Gupta M: Deficiency of cardiomyocytespecific microRNA-378 contributes to the development of cardiac fibrosis involving a transforming growth factor beta (TGFbeta1)-dependent paracrine mechanism. J Biol Chem 2014;289:27199-27214.

17 Hyun J, Wang S, Kim J, Rao KM, Park SY, Chung I, Ha CS, Kim SW, Yun YH, Jung Y: MicroRNA-378 limits activation of hepatic stellate cells and liver fibrosis by suppressing Gli3 expression. Nat Commun 2016;7:10993.

18 Yao QY, Xu BL, Wang JY, Liu HC, Zhang SC, Tu CT: Inhibition by curcumin of multiple sites of the transforming growth factor-beta1 signalling pathway ameliorates the progression of liver fibrosis induced by carbon tetrachloride in rats. BMC Complement Altern Med 2012;12:156.

19 Weiskirchen R, Gressner AM: Isolation and culture of hepatic stellate cells. Methods Mol Med 2005;117:99113.

20 Bertolino P, Trescol-Biemont MC, Rabourdin-Combe C: Hepatocytes induce functional activation of naive CD8+ T lymphocytes but fail to promote survival. Eur J Immunol 1998;28:221-236.

21 Zheng J, Wu C, Lin Z, Guo Y, Shi L, Dong P, Lu Z, Gao S, Liao Y, Chen B, Yu F: Curcumin up-regulates phosphatase and tensin homologue deleted on chromosome 10 through microRNA-mediated control of DNA methylation--a novel mechanism suppressing liver fibrosis. FEBS J 2014;281:88-103.

22 Waidmann O, Bihrer V, Pleli T, Farnik H, Berger A, Zeuzem S, Kronenberger B, Piiper A: Serum microRNA-122 levels in different groups of patients with chronic hepatitis B virus infection. J Viral Hepat 2012;19:e58-65.

23 Schmittgen TD, Livak KJ: Analyzing real-time PCR data by the comparative C(T) method. Nat Protoc 2008;3:1101-1108.

24 Ogawa T, Enomoto M, Fujii H, Sekiya Y, Yoshizato K, Ikeda K, Kawada N: MicroRNA-221/222 upregulation indicates the activation of stellate cells and the progression of liver fibrosis. Gut 2012;61:1600-1609. 


\section{Cellular Physiology Cell Physiol Biochem 2016;39:2409-2420 \begin{tabular}{ll|l} 
DOI: 10.1159/000452509 & $\begin{array}{l}\text { O 2016 The Author(s). Published by S. Karger AG, Basel } \\
\text { www.karger.com/cpb }\end{array}$
\end{tabular} \\ Yu et al.: MicroRNA-378a-3p in Hepatic Stellate Cell}

25 Wang B, Li W, Guo K, Xiao Y, Wang Y, Fan J: miR-181b promotes hepatic stellate cells proliferation by targeting p27 and is elevated in the serum of cirrhosis patients. Biochem Biophys Res Commun 2012;421:4-8.

26 Lin J, Chen A: Activation of peroxisome proliferator-activated receptor-gamma by curcumin blocks the signaling pathways for PDGF and EGF in hepatic stellate cells. Lab Invest 2008;88:529-540.

27 Li LH, Gao Q Wang XY, Guo Z]: [miR-378 suppresses HBV-related hepatocellular carcinoma tumor growth by directly targeting the insulin-like growth factor 1 receptor]. Zhonghua Gan Zang Bing Za Zhi 2013;21:609-613.

28 Bellafante E, Murzilli S, Salvatore L, Latorre D, Villani G, Moschetta A: Hepatic-specific activation of peroxisome proliferator-activated receptor gamma coactivator-1beta protects against steatohepatitis. Hepatology 2013;57:1343-1356.

29 Huang N, Wang J, Xie W, Lyu Q, Wu J, He J, Qiu W, Xu N, Zhang Y: MiR-378a-3p enhances adipogenesis by targeting mitogen-activated protein kinase 1. Biochem Biophys Res Commun 2015;457:37-42.

30 Jeon TI, Park JW, Ahn J, Jung CH, Ha TY: Fisetin protects against hepatosteatosis in mice by inhibiting miR378. Mol Nutr Food Res 2013;57:1931-1937.

31 Nagalingam RS, Sundaresan NR, Gupta MP, Geenen DL, Solaro RJ, Gupta M: A cardiac-enriched microRNA, miR-378, blocks cardiac hypertrophy by targeting Ras signaling. J Biol Chem 2013;288:11216-11232.

32 Nguyen HC, Xie W, Yang M, Hsieh CL, Drouin S, Lee GS, Kantoff PW: Expression differences of circulating microRNAs in metastatic castration resistant prostate cancer and low-risk, localized prostate cancer. Prostate 2013;73:346-354.

33 Fedorko M, Stanik M, Iliev R, Redova-Lojova M, Machackova T, Svoboda M, Pacik D, Dolezel J, Slaby O: Combination of MiR-378 and MiR-210 Serum Levels Enables Sensitive Detection of Renal Cell Carcinoma. Int J Mol Sci 2015;16:23382-23389.

34 Liu H, Zhu L, Liu B, Yang L, Meng X, Zhang W, Ma Y, Xiao H: Genome-wide microRNA profiles identify miR378 as a serum biomarker for early detection of gastric cancer. Cancer Lett 2012;316:196-203.

35 Clevers H: Wnt/beta-catenin signaling in development and disease. Cell 2006;127:469-480.

36 Kim TH, Kim SH, Seo JY, Chung H, Kwak HJ, Lee SK, Yoon HJ, Shin DH, Park SS, Sohn JW: Blockade of the Wnt/beta-catenin pathway attenuates bleomycin-induced pulmonary fibrosis. Tohoku J Exp Med 2011;223:45-54.

37 Yu F, Lu Z, Huang K, Wang X, Xu Z, Chen B, Dong P, Zheng J: MicroRNA-17-5p-activated Wnt/beta-catenin pathway contributes to the progression of liver fibrosis. Oncotarget 2016;7:81-93.

38 Kordes C, Sawitza I, Haussinger D: Canonical Wnt signaling maintains the quiescent stage of hepatic stellate cells. Biochem Biophys Res Commun 2008;367:116-123.

39 Jiang F, Parsons CJ, Stefanovic B: Gene expression profile of quiescent and activated rat hepatic stellate cells implicates Wnt signaling pathway in activation. J Hepatol 2006;45:401-409.

40 Guo Y, Xiao L, Sun L, Liu F: Wnt/beta-catenin signaling: a promising new target for fibrosis diseases. Physiol Res 2012;61:337-346. 